\section{Efecto sedante del midazolam genérico versus innovador en ratas Wistar}

\section{RESUMEN}

Antecedentes: ocho de cada diez pacientes en Terapia Intensiva del Instituto Nacional de Pediatría no obtienen el mismo efecto ansiolítico y sedante con midazolam genérico $\left(\mathrm{PiSA}^{\circledR}\right)$, que con el innovador (Dormicum, Roche $^{\circledR}$ ) a pesar de que su biodisponibilidad es de 100\%.

Objetivo: determinar diferencias significativas en el efecto sedante del midazolam genérico y del innovador administrados parenteralmente.

Material y métodos: estudio aleatorizado cruzado en 24 ratas Wistar macho distribuidas en 4 grupos $(n=6)$. A cada individuo se le administró una dosis de $0.5 \mathrm{mg} / \mathrm{kg}$ de peso vía intraperitoneal. Se determinaron los grados de sedación mediante la escala de Salamone. Se midió la concentración del fármaco en las ampolletas de ambas marcas por cromatografía líquida de alta resolución.

Resultados: el efecto sedante del midazolam apareció al mismo tiempo y tuvo la misma duración, independientemente de la marca. El efecto tiende a ser más duradero con el innovador pero sin ser estadísticamente significativo (ANOVA, $p \leq 0.05$ ). Asimismo, la mayoría de los animales llegaron al nivel 3 de sedación con ambas marcas.

Conclusión: tanto el midazolam innovador como el genérico tienen el mismo efecto sedante: aparece al mismo tiempo y tiene la misma duración.

Palabras clave: midazolam, genérico, innovador, efecto sedante, ratas Wistar, terapia intensiva.

\section{Sedative effect of innovative midazolam vs. generic in Wistar rats}

\begin{abstract}
Background: Eight out of ten patients in the Intensive Care Unit of the Instituto Nacional de Pediatría do not set the same anxiolytic and sedative effect using generic midazolam $\left(\mathrm{PiSA}^{\circledR}\right)$ as with the innovative brand (Dormicum, Roche ${ }^{\circledR}$ ), although its bioavailability is $100 \%$.

Objective: To determine significant differences in the sedative effect induced by generic and by innovative midazolam given parenterally.

Materials and methods: Randomised crossover study in 24 male Wistar rats allocated into 4 groups $(n=6)$. Each individual was given a dose
\end{abstract}

Radamés Alemón-Medina ${ }^{1}$ Liliana Rivera-Espinosa ${ }^{1}$ Patricia Zárate-Castañón ${ }^{2}$ Carmen Flores-Pérez ${ }^{1}$

${ }^{1}$ Laboratorio de Farmacología.

2 Jefa del Servicio de Terapia Intensiva. Instituto Nacional de Pediatría, México.

Recibido: 15 de abril del 2014

Aceptado: 29 de septiembre del 2015

Correspondencia: Dr. Radamés Alemón Medina Laboratorio de Farmacología Instituto Nacional de Pediatría Insurgentes Sur 3700-C CP 04530, México, D.F. Tel/Fax. 525510840900 ext. 1459 ranapez@hotmail.com

Este artículo debe citarse como Alemón-Medina R, Rivera-Espinosa L, Zárate-Castañón P, Flores-Pérez C. Efecto sedante del midazolam genérico versus innovador en ratas Wistar. Acta Pediatr Mex 2015;36:434-441. 
of $0.5 \mathrm{mg} / \mathrm{kg}$ intraperitoneally; sedation levels were determined by the scale of Salamone's scale. Drug concentration in the vials of both brands was determined by high performance liquid chromatography (HPLC).

Results: The sedative effect of midazolam appeared at the same time for both groups and had the same duration regardless of the brand. The effect tends to be more durable with the innovative, but without statistical significance (ANOVA, $p \leq 0.05$ ). Likely, most animals reached Salamone's sedation level 3 with both brands.

Conclusion: Both innovative and generic midazolam brands provide the same level of sedative effect, which appears and lasts equally.

Keywords: Midazolam, generic, innovative, sedative effect, Wistar rats, intensive care unit.

\section{INTRODUCCIÓN}

El midazolam es una benzodiacepina utilizada como ansiolítico e inductor anestésico en niños atendidos en unidades de cuidados intensivos. ${ }^{1}$ Facilita la acción inhibidora del ácido gamma-aminobutírico ${ }^{2}$ y tiene efectos sedante, ansiolítico, anticonvulsivante y amnésico. Su acción farmacológica aparece muy rápidamente y el efecto dura de 10 a 30 minutos, dependiendo de la dosis. ${ }^{3,4}$ Debido a su baja toxicidad tiene un amplio espectro terapéutico; puede administrarse incluso a dosis altas para alcanzar el efecto sedante sin causar toxicidad. ${ }^{3,5}$ Asimismo, el acceso a las presentaciones genéricas de este fármaco lo hace un modelo de sedación efectivo y barato.

A pesar de que no se ha llevado a cabo un ensayo clínico controlado durante la práctica clínica del Servicio de Terapia Intensiva del Instituto Nacional de Pediatría, se ha tenido frecuentemente la apreciación de que la mayoría de pacientes que reciben midazolam genérico (Laboratorios $\mathrm{PiSA}^{\circledR}$
$200 \mu \mathrm{g} / \mathrm{kg} / \mathrm{h}$ ) no experimenta sedación adecuada por lo que se requiere aumentar la dosis (hasta $1500 \mu \mathrm{g} / \mathrm{kg} / \mathrm{h}$ ). En cambio, con el medicamento innovador (Dormicum ${ }^{\circledR}$, Laboratorios Roche) se alcanza sedación adecuada con dosis de $200 \mu \mathrm{g} / \mathrm{kg} / \mathrm{h}$.

Hasta el momento no se ha descrito la comparación entre el efecto sedante de dos marcas comerciales de midazolam en niños mexicanos atendidos en Unidades de Terapia Intensiva, lo cual es difícil debido a factores como la variabilidad genética de la población y las distintas situaciones clínicas de los pacientes. Es por ello que en el presente trabajo se ha propuesto evaluar rápidamente, en un modelo animal, el grado de sedación de las dos marcas de midazolam empleadas en los pacientes. El estudio incluye algunos factores como el análisis del contenido, la dosis, el tiempo que tarda en tener efecto y el grado de sedación en ratas Wistar. Todo con el fin de tener una primera aproximación de si, efectivamente, hay diferencia de efecto sedante debido a la marca del medicamento. 


\section{MATERIALES Y MÉTODOS}

\section{Reactivos}

Todos los reactivos fueron grado analítico o cromatografía líquida de alta resolución. El estándar de midazolam y el fosfato de potasio monobásico fueron de Sigma (MO, USA), acetonitrilo de J.T. Baker (MO, USA) y metanol de Merck $^{\circledR}$ (Darmstad, Germany). Las ampolletas de midazolam en solución intravenosa inyectable fueron de la marca Dormicum ${ }^{\circledR}$ de Laboratorios Roche (15 mg/3 mL número de lote RJ0505) y el genérico fue midazolam de los Laboratorios Pisa $^{\circledR}$ (15 mg/3 mL número de lote B053656). El agua grado cromatografía líquida de alta resolución se obtuvo con un sistema de filtración Milli-Q (Millipore, Bedford, MA, USA).

\section{Estudio aleatorizado cruzado}

Se emplearon 24 ratas Wistar macho de 16 semanas de edad, peso corporal $500 \pm 58 \mathrm{~g}$. Los animales se distribuyeron en cuatro grupos de seis individuos cada uno y se les administró midazolam de una u otra marca de forma aleatoria. El estudio se realizó en dos fases: en la primera los grupos I y II recibieron el fármaco innovador (Dormicum ${ }^{\circledR}$ ) y los grupos III y IV el genérico (midazolam de $\mathrm{PiSA}^{\circledR}$ ). Después de un período de lavado, de una semana, en la segunda fase se invirtió el orden de administración de las marcas, de modo que los grupos I y II recibieron el genérico y los grupos III y IV el innovador. Con esto se ejecutó un estudio totalmente aleatorio y cruzado en el que cada individuo fungió como su propio control. El estudio se hizo en el Bioterio de la Torre de Investigación del Instituto Nacional de Pediatría, con estricto apego a la Norma Oficial Mexicana NOM-062-ZOO-19996 de buenas prácticas de cuidado y uso de animales de laboratorio.

\section{Administración del fármaco}

Se diluyó midazolam a partir de la solución en la ampolleta de cada marca $(5 \mathrm{mg} / \mathrm{mL})$ con solución salina isotónica estéril hasta una concentración final de $2 \mathrm{mg} / \mathrm{mL}$. A cada rata se le administró (vía intraperitoneal) el volumen (en mililitros) correspondiente a $0.5 \mathrm{mg} / \mathrm{kg}$ de peso corporal.

\section{Grado de sedación}

Para vigilar los grados de sedación se empleó la escala propuesta por Salamone y sus colaboradores $^{7}$ que consta de seis etapas de sedación en orden numérico descendente; a saber:

Etapa 5: Despierto y activo. Locomoción, desplazamiento y hábitos sin alteración.

Etapa 4: Despierto e inactivo. Locomoción y hábitos disminuidos de intensidad.

Etapa 3: Sedación leve. Ojos abiertos, muy poco movimiento pero conserva su postura y responde a estímulos táctiles.

Etapa 2: Sedación moderada. Ojos a medio cerrar, pérdida total de postura y no responde a estímulos táctiles.

Etapa 1: Sedación profunda. Apenas abre los ojos, pérdida total de postura y movimiento. Sólo responde a estímulos dolorosos.

Etapa 0: Sueño. Completamente dormido.

La acción del midazolam comenzó a medirse desde el momento en que se hicieron evidentes los primeros esbozos del efecto sedante; es de- 
cir, cuando cada animal llegó a la etapa 4 de la escala de sedación.

\section{Determinación de uniformidad de contenido de las ampolletas}

Para descartar que las posibles diferencias en el efecto sedante se debieran a alteraciones en las concentraciones del principio activo se determinó la uniformidad de contenido de las ampolletas de midazolam de ambas marcas por medio de cromatografía líquida de alta resolución. De un lote de diez ampolletas de cada marca se tomaron tres al azar. Se hizo la inspección visual del contenido y se determinó la hermeticidad del envase, así como la presencia o ausencia de partículas en suspensión. Posteriormente se vació el contenido en un tubo de vidrio estéril y se determinó su pH, más tarde se realizó una dilución 1:1,000 en agua estéril para obtener una concentración de $5 \mu \mathrm{g} / \mathrm{mL}$ que fue analizada.

Se utilizó un equipo de cromatografía líquida que consta de una bomba cuaternaria modelo 515, un inyector automático modelo 717 , un detector UVNis modelo 2487, todo de la marca Waters (Milford, MA, USA). Para la determinación del contenido se utilizo un método previamente validado en nuestro laboratorio, con una columna Pursuit C18 $5 \mu \mathrm{m}$ de $150 \times 3.9$ mm marca Agilent ${ }^{\circledR}$. Se utilizó una fase móvil de buffer de fosfatos $\mathrm{pH} 4.4$ y acetonitrilo (70:30 v/v) a un flujo de $1 \mathrm{~mL} / \mathrm{min}$, las muestras se detectaron a una longitud de onda de $220 \mathrm{~nm}$. El análisis de las muestras se hizo por sextuplicado en un espectro de concentraciones de curva de 0.1 a $10 \mu \mathrm{g} / \mathrm{mL}$, con un valor de $r^{2}=0.999$. El fármaco se cuantificó por un método analítico previamente validado 8 y fue lineal, preciso, exacto y selectivo para midazolam entre otros agentes sedantes y analgésicos de posible administración concomitante. Los coeficientes de variación de todos los parámetros de validación fueron menores a 5\% y la variación con respecto al valor nominal fue de $3.5 \%$.

\section{Análisis estadístico}

Los resultados de tiempo de aparición de acción y duración del efecto se expresan en promedio y desviación estándar (media $\pm \mathrm{DE}$ ) y se procesaron mediante análisis de varianza de un solo sentido (ANOVA, $p \leq 0.05$ ).

\section{RESULTADOS}

No obstante que con la marca innovadora algunos animales tuvieron un efecto más duradero que con el genérico (*grupos I y IV) el análisis de ANOVA de un solo factor $(p \leq 0.05)$ demostró que no hay diferencias significativas entre la aparición de la acción ni en la duración del efecto sedante con respecto a la marca (Cuadro 1 ). Con respecto al grado de sedación de la escala de Salamone, a las dosis administradas, todos los individuos tratados alcanzaron la etapa 3: conservaron su postura y tenían los ojos abiertos pero no tenían locomoción ni desplazamiento. La Figura 1 muestra los porcentajes de individuos en cada grupo que alcanzaron etapas más profundas de sedación ( 2 y 1 ). Ningún individuo se quedó completamente dormido (etapa 0). En los grupos I y III el midazolam genérico logró que un mayor porcentaje de animales alcanzara etapas ulteriores de sedación. En el grupo II ambas marcas obtuvieron el mismo porcentaje de animales en etapa 3 de la escala de Salamone. El grupo IV

Cuadro I. Comparación de la aparición del efecto sedante y su duración en ratas Wistar tratadas con midazolam innovador $\left(\right.$ Dormicum $\left.^{\circledR}\right)$ y genérico (Midazolam, Pisa ${ }^{\circledR}$ )

\begin{tabular}{lcccc}
\hline $\begin{array}{c}\text { Grupo } \\
(\mathbf{N}=6)\end{array}$ & $\begin{array}{c}\text { Aparición de acción } \\
(\mathbf{m i n})\end{array}$ & \multicolumn{2}{c}{$\begin{array}{c}\text { Duración del efecto } \\
(\mathbf{m i n})\end{array}$} \\
& Innovador & Genérico & Innovador & Genérico \\
I & $3.8 \pm 2.7$ & $2 \pm 0.8$ & $7.8 \pm 7.2 *$ & $3.8 \pm 2$ \\
II & $2.2 \pm 1.1$ & $1.6 \pm 0.9$ & $3.8 \pm 1.6$ & $2.8 \pm 1.8$ \\
II & $2.6 \pm 0.8$ & $2 \pm 0.5$ & $3.3 \pm 0.8$ & $4.5 \pm 1.8$ \\
IV & $2.4 \pm 1.2$ & $2.4 \pm 1.1$ & $11.5 \pm 9.6 *$ & $4.4 \pm 3.1$
\end{tabular}

*Valores sugerentes de un efecto más duradero con el innovador comparado con el genérico pero sin significación estadística (ANOVA, $p \leq 0.05$ ). 
tratado con el genérico tuvo $84 \%$ de animales en etapas 2 y 1 de sedación; fue el único grupo donde el genérico obtuvo grados de sedación más profunda que el innovador (Figura 1).

En la Figura 2 muestra los cromatogramas de ambas marcas: no hubo diferencias, lo que indica que las ampolletas que fabrican ambos laboratorios tienen la misma cantidad de fármaco. Asimismo, dado que se trata de un fármaco de administración intravenosa en humanos la biodisponibilidad (F) es de 100\% para ambas marcas. Cabe mencionar que la vía intraperitoneal empleada en este trabajo también origina una biodisponibilidad de 100\% (Figura 2).

\section{DISCUSIÓN}

\section{Aparición de acción}

El efecto sedante del midazolam comenzó a los 2 minutos, en promedio, independientemente de la marca; con el innovador (Dormicum ${ }^{\circledR}$ ) el

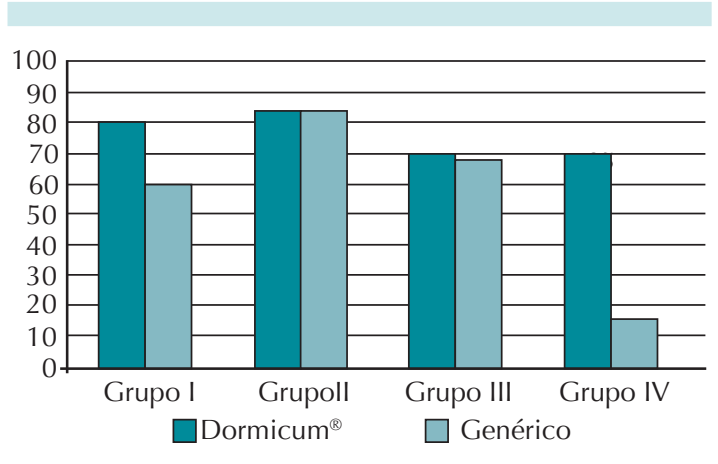

Figura 1. Porcentajes de animales, de cada grupo, que alcanzaron el nivel de sedación 3 de la escala de Salamone. Las barras oscuras representan a Dormicum ${ }^{\circledR}$ de Laboratorios Roche ${ }^{\circledR}$ y las claras al midazolam de Laboratorios Pisa ${ }^{\circledR}$. En todos los grupos hubo al menos un individuo que alcanzó el nivel 3 de sedación independientemente de la marca. En el grupo IV la mayoría de los animales alcanzaron niveles más profundos de sedación a la misma dosis de medicamento genérico (niveles 2 y 1 ).

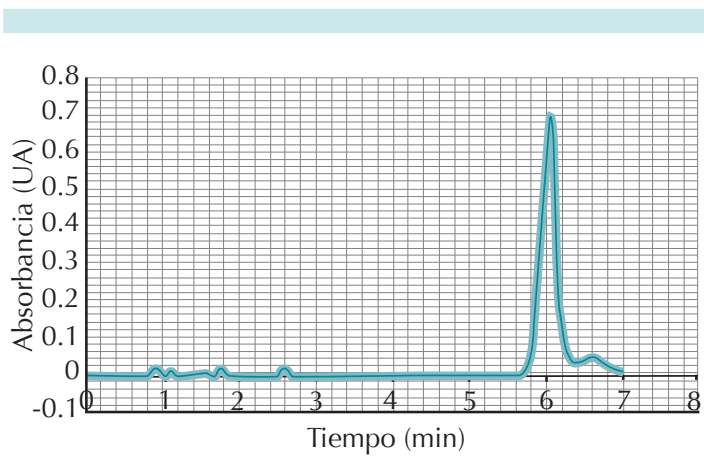

Figura 2. Cromatograma que muestra el tiempo de retención (6.1 minutos) del midazolam de ambas marcas. La línea oscura es de Dormicum ${ }^{\circledR}$ y la clara del midazolam de Pisa ${ }^{\circledR}$. Ambos perfiles se superponen perfectamente, lo que indica uniformidad de contenido en ambas marcas.

efecto apareció a los 2.2 minutos en el grupo II, mientras que en el grupo I apareció a los 3.8 minutos (Cuadro 1). Dichos tiempos de aparición del efecto fueron el mínimo y el máximo, respectivamente, para esta marca comercial. El efecto sedante del midazolam genérico $\left(\mathrm{PiSA}^{\circledR}\right)$ apareció también a los 2 minutos. En el grupo II el efecto apareció inclusive antes que con la marca innovadora (1.6 vs. $2.2 \mathrm{~min}$ ); no obstante, dichas diferencias no fueron estadísticamente significativas en la prueba ANOVA de un solo sentido $(F=3.64$ vs. F crítica $=4.06)$.

Con el diseño experimental empleado en este trabajo se ha descartado la influencia de la condición de enfermedad o daño orgánico del individuo, así como de la variabilidad genética de la población pediátrica dado que las ratas Wistar a las que se les administró el fármaco son animales sanos, con la misma alimentación y viviendo en las mismas condiciones; asimismo, su variabilidad genética es muy baja ya que todos resultaron de la misma generación filial. Por todo esto se puede sugerir que las diferencias en el efecto sedante de ambas marcas no se deben a la formulación química de las mismas. En este 
sentido se ha tenido una primera etapa que habrá de confirmarse con un ensayo clínico controlado y comparándolo con marcadores genéticos del metabolismo del midazolam como el citocromo CYP3A4.

\section{Duración del efecto}

Los datos del Cuadro 1 muestran que los efectos sedantes inducidos por el midazolam de ambas marcas duraron, en promedio, $6.6 \mathrm{mi}-$ nutos el innovador y 3.8 minutos el genérico; lo suficiente para reducir la ansiedad e inducir la anestesia antes de realizar intervenciones quirúrgicas. La duración del efecto, lo mismo que su aparición, comenzaron a contarse en cuanto cada animal mostró los primeros indicios de pérdida de equilibrio y desorientación, terminó cuando el animal recuperó su posición erguida y sus instintos naturales de exploración táctil y olfativa.

En los grupos I y IV el efecto sedante del Dormicum $^{\circledR}$ fue, en promedio, más duradero que en los grupos II y III con el fármaco genérico; el efecto duró 7.8 y 11.5 minutos en promedio, respectivamente (Cuadro 1). Estos datos sugieren que hay una ligera tendencia a que el efecto sedante del midazolam innovador sea más duradero en algunos individuos. De hecho, en algunos animales de los grupos I y IV el efecto duró hasta 25 o 30 minutos; sin embargo, no todos los individuos en estos mismos grupos tuvieron una sedación tan prolongada; al promediar los tiempos y ejecutar el análisis estadístico las diferencias no fueron estadísticamente significativas: $\mathrm{F}=3.32$ $v s$. F crítica $=4.06$.

El hecho de que con el midazolam de la marca innovadora la duración de la sedación haya sido mayor que con el genérico en algunos individuos podría deberse a la variabilidad de respuesta al fármaco que, incluso entre sujetos con gran semejanza genética, es muy evidente.

\section{Grado de sedación}

A la dosis empleada en este trabajo 70 a $80 \%$ de los individuos que recibieron Dormicum ${ }^{\circledR}$ vía intraperitoneal alcanzaron el grado 3 de la escala de sedación de Salamone; es decir, tuvieron sedación leve, con los ojos abiertos y muy poco movimiento aunque conservando su postura. El individuo, además, se quedó quieto pero respondía a estímulos táctiles. Sólo 20 a $30 \%$ de los animales tratados con midazolam de esta misma marca, a esta dosis, alcanzaron grado 2 de sedación, más profunda que el resto.

El comportamiento de los animales tratados con midazolam genérico fue prácticamente el mismo que con el innovador: los grupos I, II y III tuvieron 60 a $80 \%$ de los individuos en el grado 3 y el resto en grado 2. La única variante fue en el grupo IV donde la mayoría (52\%) de los animales alcanzó el grado de sedación 1; 32\% el grado 2 y sólo $16 \%$ el grado 3 ; es decir, que el midazolam genérico obtuvo una sedación más profunda en este grupo. No obstante, las diferencias tampoco fueron estadísticamente significativas.

\section{Implicaciones clínicas}

Del presente estudio se desprende que en individuos genéticamente idénticos, tal como este modelo de ratas Wistar, el efecto sedante de ambas marcas de midazolam aparece al mismo tiempo. Asimismo, aunque con el midazolam innovador el efecto tendió a ser más duradero, no pudo considerarse como una diferencia real entre las marcas. Adicionalmente, la uniformidad de contenido entre las ampolletas de ambas marcas (Figura 2) mostró que ambas marcas contienen exactamente la misma cantidad de medicamento.

Es posible sugerir que las diferencias observadas en el servicio de terapia intensiva podrían deberse a diferencias individuales de respuesta 
al fármaco o a la condición clínica de cada paciente. Sabemos que la farmacocinética del midazolam en pacientes críticamente enfermos puede modificarse como resultado de una o varias insuficiencias orgánicas, ${ }^{9}$ sobre todo las disfunciones hepática o renal. ${ }^{10-12}$ Por otro lado, en pacientes adultos la eliminación del midazolam varía notablemente entre sanos y enfermos; aunque en pacientes pediátricos no se han encontrado dichas variaciones es de esperarse que también ocurran. ${ }^{13-15} \mathrm{Un}$ aspecto adicional que puede influir en las diferencias observadas en la clínica es la administración concomitante de inhibidores del CYP3A (cimetidina, eritromicina, claritromicina, diltiazem, verapamilo). ${ }^{16-18}$

Finalmente, es importante señalar que las diferencias sedantes del midazolam de distintos fabricantes podrían deberse a una apreciación subjetiva del personal. Respecto del midazolam genérico empleado en Latinoamérica se ha publicado que: "no se evidencia una verdadera presencia de falla terapéutica con el uso de midazolam genérico comparado con el innovador" ${ }^{19}$ Sería conveniente realizar un estudio clínico controlado y aleatorio en niños mexicanos para descartar verdaderamente la influencia de la marca del fármaco o para adjudicarla sólo a la variabilidad individual.

\section{Implicaciones farmacoeconómicas}

Desde el punto de vista económico existe preocupación del personal de enfermería en la unidad de cuidados intensivos de nuestra institución: si los pacientes requieren dosis mayores de midazolam genérico para lograr sedación ello implica el uso de 10 ampolletas de genérico por cada ampolleta de innovador. El costo del midazolam innovador, para nuestra institución, es de aproximadamente $\$ 750.00$ pesos (caja con cinco ampolletas de $15 \mathrm{~mL}$ ) mientras que el costo del genérico es de aproximadamente $\$ 50.00$ pesos (caja con cinco ampolletas de
$15 \mathrm{~mL}$ ). De modo que si se tuviera que aplicar diez veces más cantidad de midazolam genérico para obtener el mismo efecto que con el de innovador el costo total sería de $\$ 500.00$ pesos; aún así sería más económico. No obstante, también hay que considerar que a mayores dosis de medicamento existen también más probabilidades de interacción farmacológica y de reacciones adversas; sobre todo en pacientes que reciben varios medicamentos en terapia intensiva.

\section{CONCLUSIÓN}

En nuestro modelo biológico de ratas Wistar (sanas y de la misma generación filial), bajo las condiciones experimentales y la escala de sedación empleadas se ha mostrado que, exceptuando sutiles diferencias ambas marcas de midazolam (innovador y genérico) tienen el mismo efecto sedante, que aparece al mismo tiempo y que tiene la misma duración. Se sugiere llevar a cabo un estudio clínico controlado y aleatorio, con población pediátrica mexicana representativa, para descartar la influencia de la marca del fármaco en sus resultados clínicos.

\section{REFERENCIAS}

1. Hartwig S, Roth B, Theisohn M. Clinical experience with continuous intravenous sedation using midazolam and fentanyl in the paediatric intensive care unit. European Journal of Pediatrics 1991;150:784-788.

2. Lancel M, Crönlein T, Faulhaber J. Role of GABAA receptors in sleep regulation differential effects of muscimol and midazolam on sleep in rats. Neuropsychopharm 1996;15:63-74.

3. Katzung BG, Basic \& Clinical Pharmacology, 10th edition, New York, USA, 1995; 1182.

4. Reves JG, Fragen RJ, Vinik HR, et al. Midazolam: pharmacology and use. Anesthesiology 1985;62:310-24.

5. Heizmann P, Eckert M, Ziegler WH. Pharmacokinetics and bioavailability of midazolam in man. $\mathrm{Br} \mathrm{J}$ Clin Pharmacol 1983;16(Suppl 1):43S-49S.

6. NOM-062-ZOO-1999. Especificaciones técnicas para la producción, cuidado y uso de los animales de laboratorio. Diario Oficial de la Federación. México. 2001. 
7. Chuck TL, McLaughlin PJ, Arizzi-LaFrance MN, Salamone JD, Correa M. Comparison between multiple behavioral effects of peripheral ethanol administration in rats: Sedation, ataxia, and bradykinesia. Life Sci 2006;79:154-161.

8. Flores-Pérez C, Chávez-Pacheco JL, Flores-Pérez J, JuárezOlguín H, Ramírez-Mendiola B, García-Álvarez R, GonzálezZamora JF, Changin-Guerra A. Development and Validation of a Method to Quantify Midazolam in a New Oral Formulation for Pediatric Use. AJAC 2012;3:552-558.

9. Shelly MP, Mendel L, Park GR. Failure of critically ill patients to metabolize midazolam. Anaesth 1987;42:619-26.

10. Marriot P, Laasch HU, Wilbraham L, Marriot A, England RE, Martin DF. Conscious sedation for endoscopic and non-endoscopic interventional gastrointestinal procedures: meeting patients expectations, missing the standard. Clin Radiol 2004;59(2):180-185.

11. Bauer TM, Ritz R, Haberthür C, Ha HR, Hunkeler W, Sleight AJ, Scollo-Lavizzari G, Haefeli WE. Prolonged sedation due to accumulation of conjugated metabolites of midazolam. Lancet 1995;345:145-47.

12. Vree TB, Shimoda M, Driessen JJ, et al. Decreased plasma albumin concentration results in increased volume of distribution and decreased elimination of midazolam in intensive care patients. Clin Pharmacol Ther 1989;46:537-44.
13. Park GR, Miller E, Navapurkar V. What changes drug metabolism in critically ill patients? II. Serum inhibits the metabolism of midazolam in human microsomes. Anaesth 1996;51:11-15.

14. Malacrida R, Fritz ME, Suter PM, et al. Pharmacokinetics of midazolam administered by continuous intravenous infusion to intensive care patients. Crit Care Med 1992;20:1123-26.

15. Tobias JD. Sedation and analgesia in the Pediatric Intensive Care Unit. Pediatric Annals 2005;34(8):636-45.

16. Smith MT, Eadie MJ, O'Rourke Brophy T. The pharmacokinetics of midazolam in man. Eur J Clin Pharmacol 1981;19: 271-78.

17. Dundee JW, Halliday NJ, Harper KW, et al. Midazolam: a review of its pharmacological properties and therapeutic use. Drugs 1984;28:519-543.

18. Shenfield G, Gross A. The cytochrome P450 system and adverse drug reactions. Adv Drug React Bull 1999;194:73942.

19. Serrano Espinosa VG. Estudio observacional del uso de midazolam genérico en el Instituto Oncológico Nacional de Panamá, para la sedación consciente en pacientes sometidos a gastroscopia. Barcelona, España. Mayo 2005. 